\title{
A commentary on "Surgical repair of vaginal vault prolapse; a comparison between ipsilateral uterosacral ligament suspension and sacrospinous ligament fixation-a nationwide cohort study"
}

\author{
Ghazaleh Rostaminia ${ }^{1}$ \\ Received: 28 September 2020 / Accepted: 22 October 2020 / Published online: 31 October 2020 \\ (C) The International Urogynecological Association 2020
}

This retrospective cohort study aimed to compare the ipsilateral uterosacral ligament suspension (IUSLS) and sacrospinous ligament fixation (SSLF) to treat vaginal vault prolapse based on the number of repeat surgeries. Data were retrieved from the Danish National Patient Registry (DNPR) during a 7-year period from January 12, 2010, through December 30, 2016. Previously hysterectomized women operated on with IUSLS or SSLF were included in the study. The exclusion criteria were history of malignancy, non-Danish citizens, or the operation performed < 20 times at a hospital in the time period. The primary outcome was a repeat operation for $\mathrm{POP}$ in the apical compartment within 5 years. Secondary outcomes were POP operations within 5 years in the anterior, posterior, or any compartment. Patients were followed until October 30, 2017. The IUSLS was performed bilaterally with three absorbable sutures on each side. The SSLF was performed unilaterally to the sacrospinous ligament with absorbable sutures.

In total, 744 patients were included in the study: 384 received IUSLS and 360 received SSLF. The groups were comparable according to age, BMI, smoking habits, parity, and previous caesarean sections. The group that received the IUSLS had a higher stage of prolapse and were less likely to have had surgery for prolapse prior to the suspension surgery. After 5 years, the cumulative proportion that underwent repeat surgery in the apical compartment was $6.5 \%$ after IUSLS and $21.8 \%$ after SSLF. For the anterior compartment, it was $4.1 \%$ after IUSLS and $9.5 \%$ after SSLF; for the posterior compartment, it was $4.5 \%$ after IUSLS and $8.5 \%$ after SSLF. The cumulative proportion that underwent a repeat surgery in any compartment was $12.4 \%$ for the IUSLS group and $30.6 \%$ for the SSLF group. The risk of repeat surgery in any compartment was 3.11 times higher after SSLF compared to IUSLS (CI: 2.06-4.72).

In summary, this study showed significantly higher numbers of repeat surgery due to pelvic organ prolapse after sacrospinous ligament fixation compared to ipsilateral uterosacral ligament suspension. The rate of repeat surgery within 5 years after IUSLS was comparable to that in the OPTIMAL trial (12.4\% vs. $8.5 \%$, respectively). However, the rate of repeat surgery after SSLF was considerably higher ( $30.6 \%$ vs. $4.6 \%$, respectively). While the current study concludes that the IUSLS procedure is a more durable procedure than unilateral SSLF for repairing vaginal cuff prolapse, it fails to consider the potential confounding factors such as the higher rate of recurrent prolapse prior the surgery in the SSLF group in this cohort, unilateral versus bilateral SSLF, and limited surgical experience with SSLF in Denmark. On the other hand, there is a need for innovation in native tissue repair techniques, as even for the OPTIMAL trial results, the long-term outcome is not satisfactory.

Publisher's note Springer Nature remains neutral with regard to jurisdictional claims in published maps and institutional affiliations.

Ghazaleh Rostaminia

ghazalerostaminia@yahoo.com

1 NorthShore University HealthSystem/ University of Chicago,

Skokie, IL, USA 